\title{
Pyranine Fluorescence Quenching for the Characterization of Solutions
}

\author{
Qiuxia Wang1,2, Kris A. Berglund1,3,4* \\ ${ }^{1}$ Departments of Chemical Engineering \& Materials Science, Michigan State University, East Lansing, MI, USA \\ ${ }^{2}$ Departments of Chemistry, Michigan State University, East Lansing, MI, USA \\ ${ }^{3}$ Departments of Food Science \& Human Nutrition, Michigan State University, East Lansing, MI, USA \\ ${ }^{4}$ Division of Chemical Engineering, Luleå University of Technology, Luleå, Sweden \\ Email: "berglund@msu.edu
}

Received 17 September 2015; accepted 18 January 2016; published 22 January 2016

Copyright (C) 2016 by authors and Scientific Research Publishing Inc.

This work is licensed under the Creative Commons Attribution International License (CC BY).

http://creativecommons.org/licenses/by/4.0/

(c) () Open Access

\begin{abstract}
Fluorescence quenching of pyranine by tryptophan, phenylalanine, and nicotinic acid was investigated by using steady state and time resolved fluorescence spectroscopy. On a comparative basis, nicotinic acid is a very strong quencher of pyranine fluorescence, tryptophan is a moderate quencher and phenylalanine is a weak quencher. The strong quenching is the result of the hydrogen bonding complex between pyranine and amine which existed in both tryptophan and nicotinic acid. Contact complex will form between phenylalanine and pyranine which is the reason of quenching of pyranine by phenylalanine. Associates will form in tryptophan and phenylalanine due to the zwitterion ${ }^{+} \mathrm{H}_{3} \mathrm{NRCOO}^{-}$or/and hydrogen bond. Higher concentrations favor the formation of aggregates in the supersaturated solution which made the quenching curve different from unsaturated solution dramatically.
\end{abstract}

\section{Keywords}

Fluorescence Quenching, Pyranine, Solution Structure, Tryptophan

\section{Introduction}

The majority of chemical or biological reactions occur in solution. It is clear that the properties of solute molecule will be modified by solvents. Understanding the interaction is essential for the intrinsic properties of solutes to be understood better [1]. These interactions have profound influence on crystallization from solution.

Supersaturation is an essential requirement for all crystallization operations. The formation of nuclei is the

\footnotetext{
${ }^{*}$ Corresponding author.
} 
beginning point of the crystallization. The nucleation of solute must somehow depend upon the solute molecules in a supersaturated solution and also depend upon the various molecular interactions, such as solute-solute and solute-solvent interactions. Understanding the structure of solution, especially the structure of supersaturated solutions, can help in the better design and control of crystallization.

Several studies have focused on the structure of supersaturated solutions and many techniques were applied for the research, such as NMR, X-ray diffraction and Raman spectroscopy, and some theories about the structure of supersaturated solution had been proposed [2]-[6]. Mullin and Leci suggested that solute clusters formed in a supersaturated solution of citric acid by observing an isothermal column of a supersaturated solution of citric acid which generated a concentration gradient over a period of several days. The concentration gradient developed as a result of the density difference between the clusters and the solution [2]. Cussler observed different rates between cluster diffusion and molecular diffusion in many binary systems, which supported the cluster assumption [3]. Myerson and co-workers observed the decrease in the diffusivities of urea, glycine solutions, which provided evidence for molecular aggregation [4]. Hussman et al. and McMahon et al. also confirmed cluster formation on alkali nitrate solutions by using Raman spectroscopy [5] [6]. Although such research has been conducted for decades, no general accepted theories have emerged.

All the previous work was focused on higher solubility systems whose solubility can be as high as 50 wt\%. No information about the organization of lower solubility systems in the supersaturated region has been given. Concentration gradients, the evidence for the formation of solute clusters, will not be noticeable in low solubility systems; therefore, there exists a need to find suitable techniques to study supersaturated solution structure of low solubility systems.

Fluorescence quenching is a very useful method used to study both physical phenomena and biochemical research [7]-[11]. Fluorescence quenching had been shown to be a very valuable tool to measure micelle size as well as the dynamic properties of the aggregate and of the solubilized species in the host structure [7]. Quenching studies can also be used to reveal the localization of fluorophores in proteins and membranes, and their permeability to quenchers. Fluorescence quenching requires the contact of the fluorophore and quenchers, so fluorescence quenching experiments can yield valuable information about fluorophore and quencher interactions and also reveal the accessibility of fluorophores to quenchers.

Time-resolved fluorescence represents a powerful optical spectroscopic technique for monitoring the dynamics of macromolecular interactions in solution [12]-[15]. Time-resolved fluorescence is a useful tool to study the interactions of solute and solvent. A wealth of information about the organization and dynamics of solvent-solute interactions in the microenviornment of a probe molecule can be obtained by studying the relaxation processes on a variety of time scales. Time-resolved techniques are able to directly separate and quantify the relevant fluorescence parameters that contribute to the steady-state emission parameters. The emission lifetime of a probe molecule is highly sensitive to the solvation. The use of time resolved fluorescence methods to investigate the microscopic interactions between the solvent and solute and the crystallization behavior should result in a more directed and rational approach to understand and control crystallization.

In this paper fluorescence quenching techniques in steady state and time resolved decay have been utilized to study the solution of low solubility systems. Pyranine fluorescence quenched by tryptophan, phenylalanine, and nicotinic acid by steady state and time resolved fluorescence were investigated. The quenching mechanism of pyranine fluorescence by different quenchers is studied. The solution organization in tryptophan and phenylalanine solutions and also the organization of supersaturated solution are characterized.

\section{Materials and Methods}

Pyranine, also known as FDA D \& C Green No. 8, was obtained from Lancaster, Windharn, NH and used as received. Aqueous solutions of pyranine were prepared by dissolution of $0.026 \mathrm{~g}$ in $5 \mathrm{ml}$ HPLC grade water. Solutions were prepared by dissolving the appropriate amount of solutes in $5 \mathrm{ml}$ HPLC water. Supersaturated solutions were prepared by heating the solution in a water bath until all the solute was dissolved and subsequent cooling to room temperature. The solutions were used as prepared without any degassing.

Probe molecules were introduced by addition of five microliters of the pyranine solution to $5 \mathrm{ml}$ aqueous tryptophan and phenylalanine solutions resulting in a probe concentration of $10^{-5} \mathrm{M}$ and 20 microliters of the pyranine to $5 \mathrm{ml}$ nicotinic acid aqueous solutions resulting in a probe concentration of $4 \times 10^{-5} \mathrm{M}$. Fluorescence spectra of the pyranine in the aqueous solutions were collected within two hours of the preparation of the solu- 
tions. All spectra were collected at room temperature with a Spex 1681 Fluorolog spectrometer equipped with a Xenon lamp source and a PMT detector. A quartz sample holder was used in all cases. Spectra were recorded at $1.00 \mathrm{~mm}$ increments and an integration time of $1.0 \mathrm{sec}$. An excitation wavelength of $342 \mathrm{~nm}$ for pyranine was used. The emission spectra were recorded over a range from 350 - $600 \mathrm{~nm}$ for pyranine. Monochromator slits were set at $0.5 \mathrm{~mm}$ for spectra.

Lifetime measurements of pyranine in the aqueous solutions were collected within several hours of the preparation the solutions. The lifetime was measured by using a single photon counting time correlated method with a photomultiplier tube (PMT) as the detector and a YAG laser with LDS 698 dye as excitation light source. The excitation wavelength was $342 \mathrm{~nm}$ and emission wavelength was $510 \mathrm{~nm}$. The other conditions are high voltage $3200 \mathrm{v}$, TAC range $100 \mathrm{~ns}$, power $120 \mathrm{mw} /$ cavity dump.

The absorption spectra were collected using a Perkin-Elmer Lambda Array 3840 UV/Vis Spectrophotometer. The absorption spectra of pyranine in water were collected with HPLC water as the reference and the absorption spectra of pyranine in solution of tryptophan, phenylalanine and nicotinic acid were collected by using the same concentration of tryptophan, phenylalanine and nicotinic acid solutions as references.

\section{Theory}

Fluorescence quenching involves a variety of processes, including excited state reactions, excited state proton transfer, excited state electron transfer, energy transfer, complex formation and collision quenching [16]. The quenching from diffusion-controlled encounters, the most common quenching process, is called dynamic quenching.

Dynamic quenching of fluorescence can be described by the Stern-Volmer Law [16]

$$
I_{0} / I-1=K_{s v}[Q]
$$

$I_{0}$ is initial intensity without the quencher, $I$ is emission intensity of single-emission wavelength with the quencher. $K_{s v}$ is the Stern-Volmer quenching constant and $Q$ is the concentration of quencher. If the mechanism of quenching is by dynamic quenching a straight line will result by plotting $I_{0} / I$ versus concentration of quenchers and the slope is the Stern-Volmer quenching constant. For some cases where $I_{0}$ is smaller than $I$ at low concentration, the modified SV equation shown in Equation (2) can be used. The interpretation of quenching data is based more on the shape of the curve and initial intensity $I_{0}$ will not affect the shape of the quenching curves.

$$
1 / I-1 / I_{0}=\left(K_{s v} / I_{0}\right)[Q]
$$

Static quenching is a frequent complicating factor in the analysis of dynamic quenching. Static quenching decreases the emission intensity, but has no effect on the lifetime of the unassociated excited fluorophores. In order to distinguish dynamic quenching and static quenching we use the lifetime form of Stern-Volmer Law to describe dynamic quenching

$$
\tau_{0} / \tau-1=K_{s v}[Q]
$$

$\tau_{0}$ is the lifetime of fluorophore without quencher, and $\tau$ is the lifetime with quencher.

Two plots from Equation (1) and Equation (3) should be indistinguishable if the simple diffusion quenching model holds. The discrepancies between the Stern-Volmer plots can supply useful chemical information, including situations in which the quencher absorbs intensely in the region of excitation and/or emission. Under these conditions, trivial absorption can produce unacceptable distortions of intensity quenching data unless complicated, and frequently unreliable, corrections are applied.

Static quenching can be described by a ground state complex or a sphere of action static quenching models. Ground state complex static quenching occurs as a result of the formation of a nonfluorescent ground state complex between the fluorophore and quencher. When this complex absorbs light it immediately returns to the ground state without emission of a photon [16]. Sphere of action static quenching model about static quenching is "active sphere" which is the volume around a quencher molecule. The fluorophore will be quenched without the need for a diffusion-controlled collision interaction if it is in the active interaction volume [17]. In many instances the fluorophore can be quenched both by collisions and also by static quenching. The characteristic feature of the Stern-Volmer plots in such circumstances is an upward curvature, concave towards the y axis. In the ground state complex model the modified form of Stern-Volmer law I [16] can be expressed as 


$$
I_{0} / I=\left(1+K_{s}[Q]\right)\left(1+K_{d}[Q]\right) \text { or }\left(I_{0} / I-1\right) /[Q]=K_{s}+K_{d}+K_{s} K_{d}[Q]
$$

$K_{s}$ and $K_{d}$ are the quenching constants of static and dynamic respectively. When $K_{s} \gg K_{d}$, static quenching is dominant or if $K_{d} \gg K_{s}$ dynamic quenching is dominant, the Stern-Volmer quenching plot will show a straight line. When there is no dominant quenching, which means $K_{s}$ is very close to $K_{d}$, the plot of $\left(I_{0} / I-1\right) /[Q]$ vs. $[Q]$ should be a straight line. From the slope and intercept we can get two quenching constants.

Birks [17] described the active sphere model using the modified Stern-Volmer law II

$$
\left(I_{0} / I\right) \exp (-V[Q])=1+K_{s v}[Q]
$$

$K_{s v}$ is the collision quenching constant, and $V$ is interaction volume or is called the static quenching constant which is related to the probability of finding a quencher molecule close enough to a newly formed excited state. A plot of $\left(I_{0} / I\right) \exp (V[Q])$ versus $Q$ by adjusting $V$ should result in a straight line.

Downward curvatures of the S-V plot were reported by Lakowicz [16], Eftink and Ghiron [18], Kikuchi and Sato [19] and by Pan and Cherry [20]. Negative deviations from Stern-Volmer Law are the results of multiple emitters with different quenching constants or inefficient quenching [16] [18]. Pan and Cherry pointed out that the downward is the characteristic of the particular quenching effect. Also the downward curvatures can be the results of the heterogeneity of the system or selective quenching. Such as different binding sites in large molecule make some fluorophores easier to be quenched than others [21]. The negative deviation from Stern-Volmer law can be described by modified Stern-Volmer Law III [22].

$$
I_{0} /\left(I_{0}-I\right)=\left(1 / f K_{s v}\right)(1 /[Q])+1 / f
$$

$f$ is the fraction of the fluorescence accessible to the quenching.

\section{Results and Discussion}

\subsection{The Mechanism of Quenching}

The fluorescence spectra of pyranine in tryptophan, nicotinic acid and phenylalanine exhibit no differences in shape and maxima in the presence and absence of solutes when the concentration of solutions are not very high although there is appreciable quenching in the presence of tryptophan and dramatic quenching in the presence of nicotinic acid. No new fluorescence peak is observed at longer wavelengths.

From the Stern-Volmer quenching curve of pyranine by tryptophan at $510 \mathrm{~nm}$ fitted by Equation (2) shown in Figure 1, an upward curve for tryptophan appears which indicates the coexistence of dynamic and static quenching of pyranine by tryptophan and a straight line which will only curve very slightly upward shows up for phenylalanine. Much stronger static quenching by tryptophan occurs. The quenching curves of pyranine by nicotinic acid at steady state and time resolved measurements are shown in Figure 2. At steady state the quenching shows a marked upward curve which is the result of coexistence of static and dynamic quenching. The quenching curve by lifetime measurement is a straight line which is common. Very strong static quenching exists in quenching of pyranine by nicotinic acid by the comparison of static and dynamic quenching curves. The quenching of pyranine by phenylalanine at $510 \mathrm{~nm}$ in steady state and time resolved decay fitted by Stern-Volmer law show totally different behavior which is shown in Figure 3. At steady state phenylalanine shows quenching ability to pyranine but phenylalanine will not quench fluorescence of pyranine dynamically which shows an increasing lifetime of pyranine with the increase concentration of phenylalanine in the lifetime measurement.

The formation of a transient excited state complex or exciplex as an intermediate in the fluorescence quenching process has been proposed by many workers [23]-[32]. Such as exciplex can be emissive which will present broad and structureless emission spectra at longer wavelength [24]. Exciplex is defined as a complex of simple integer stoichiometry formed between an excited molecule and one or more non-excited molecules [25]. The formation of exciplex was the result of intermolecular forces or Van der Waals interaction which have an electromagnetic origin and arise in a variety of ways such as dipole-dipole, polarizability-dipole, polarizability-polarizability and dispersion interactions. Hydrogen bonding interaction is often cited as efficient quenching mechanics by charge transfer or electron transfer via the hydrogen bond or by proton transfer [26]. The orbital overlap interactions play an important role in the correct exciplex geometry and also help to stabilize the exciplex but they cannot become a dominant factor for the formation of exciplex [25]. 


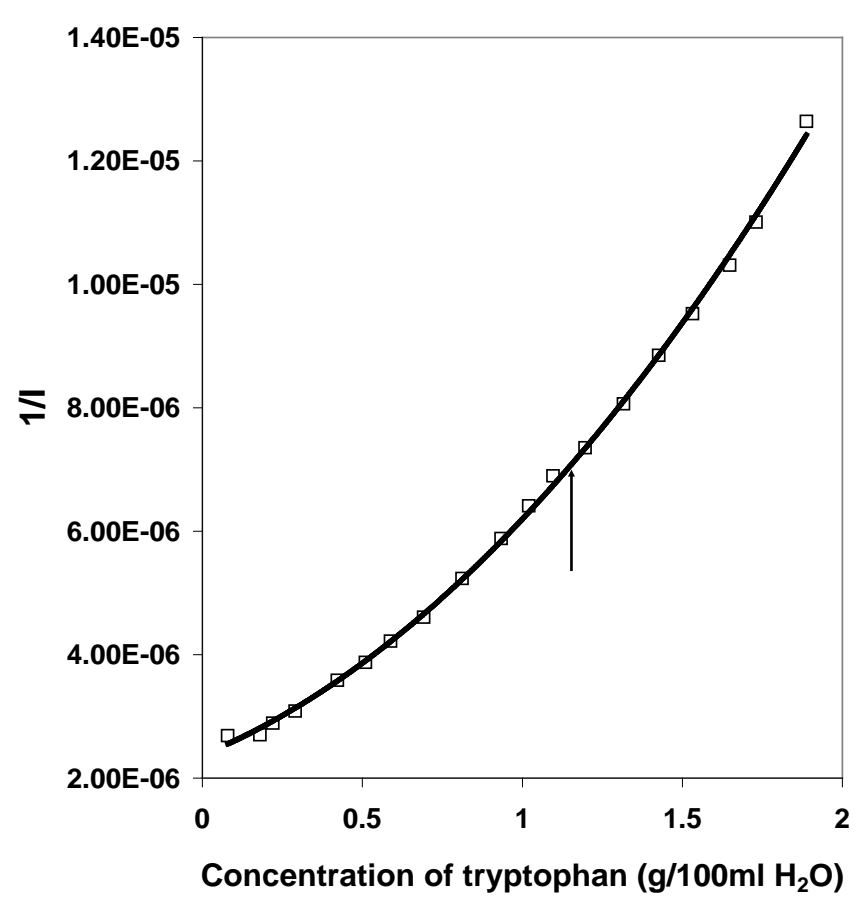

Figure 1. Quenching curve of pyranine by tryoptophan at $510 \mathrm{~nm}$ fitted by $1 / I=1 / I_{0}+\left(K_{s v} / I_{0}\right)[Q]$. Arrow indicates the solubility of tryptophan.

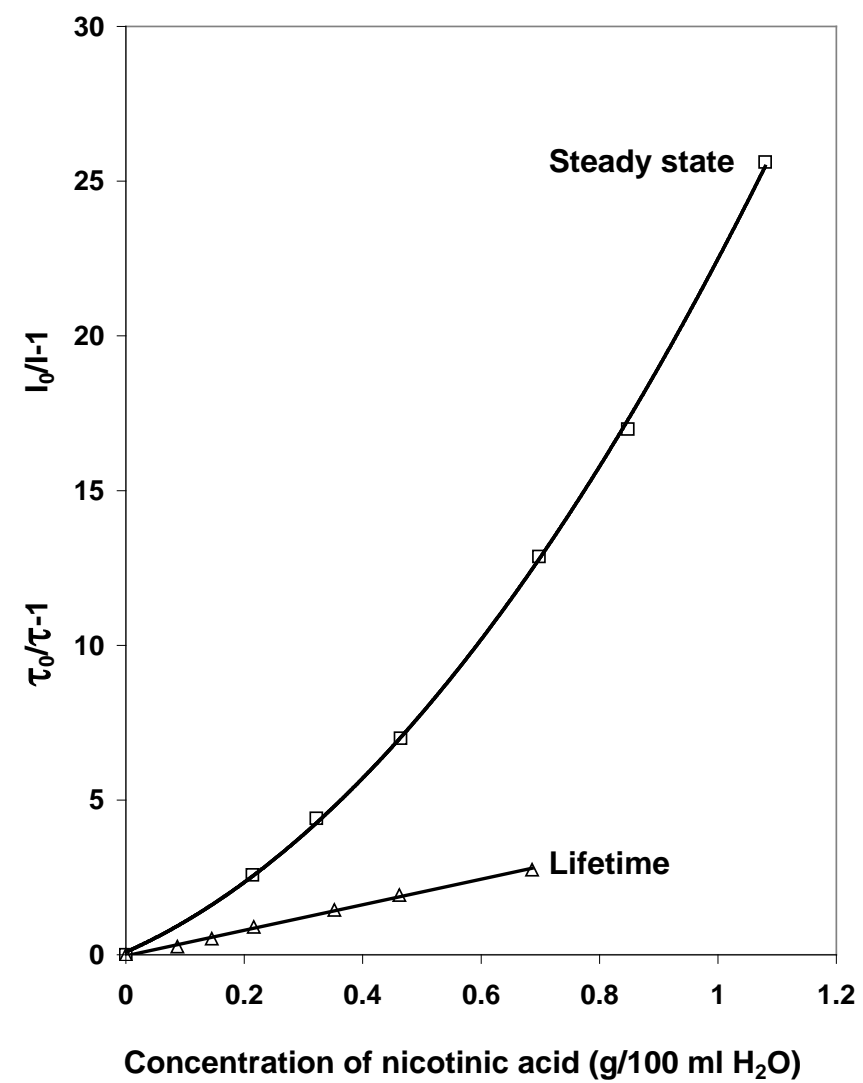

Figure 2. Quenching of pyranine by nicotinic acid at steady state and time resolved decay at $510 \mathrm{~nm}$ fitted by Stern-Volmer law. 


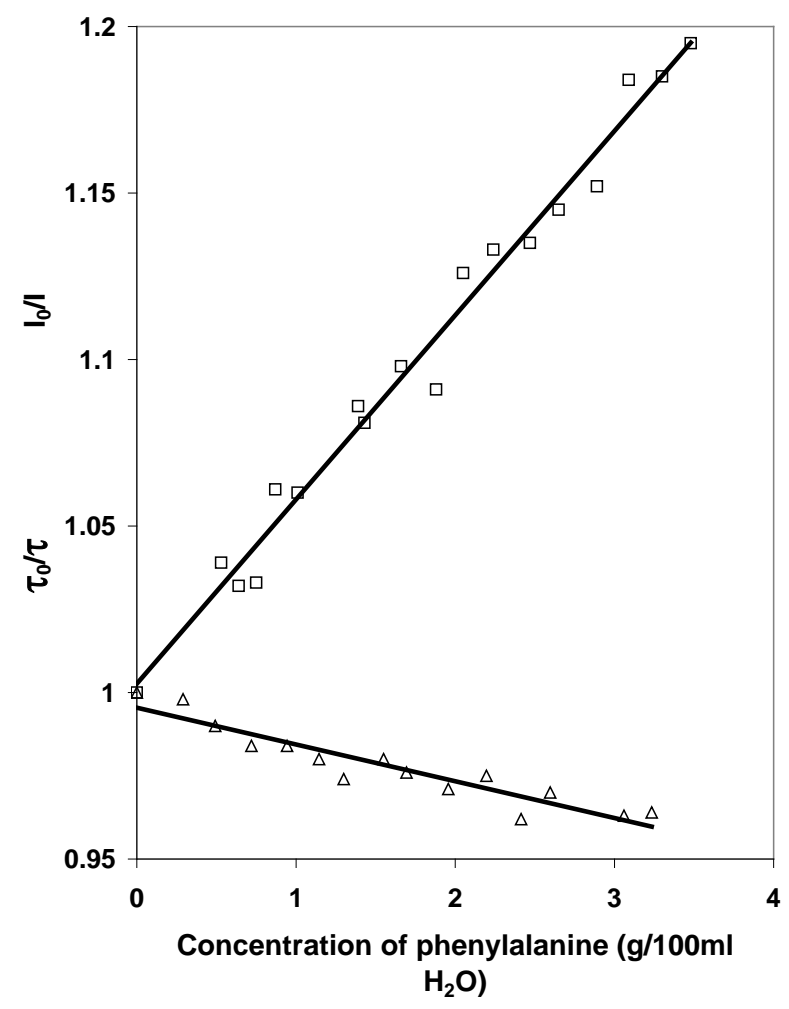

Figure 3. Quenching of pyranine by phenylalanine at steady state and time resolved decay at $510 \mathrm{~nm}$ fitted by Stern-Volmer law.

Pyridine was reported to be a strong fluorescence quencher to many fluorophores such as 1-pyrenol, 2-naphthol, 1-aminopyrene and 13H- and 7H-dibenzocarbazole which was suggested as hydrogen bonding interaction between pyranine and fluorophore in the hydrogen bonded complex [26].

The structure of tryptophan and phenylalanine are different only in the ring, one is benzene and the other is the indole ring which has a nitrogen heteroatom in five member ring. Nicotinic acid is a six member ring with one nitrogen heteroatom in the ring. Both quenching curves of pyranine by tryptophan and nicotinic acid are rather upward curves which suggest strongly that the ring nitrogen contributed to the static quenching of pyranine. Static quenching occurs either through the formation of ground state complex or by active sphere model. Amines are shown by other authors to be very efficient quenchers of aromatic molecules because they are organic electron donors [27]. Amines are good electron donors and the hydroxyl group in pyranine is a good electron acceptor. So it is reasonable to conclude that the hydrogen bonding complex will form between pyraninetryptophan and pyranine-nicotinic acid which is the reason for the strong static quenching from both nicotinic acid and tryptophan.

At the experimental conditions ( $\mathrm{pH} 7$ and low concentration of acid) pyranine ( $\mathrm{pKa}=7.5$ ) will exist as a protonated species. This protonated species will form a hydrogen bonding complex with tryptophan and nicotinic acid at ground state. When pyranine was excited the proton transfer will take place between pyranine and amine due to the low pKa (0.5) at excited states. This proton transfer process will quench the fluorescence of pyranine. The mechanism of quenching, including both the ground state complex and exciplex, is summarized in scheme shown in Figure 4. Hydrogen bonding results in more efficient quenching which is the reason of stronger quenching ability of tryptophan and nicotinic acid to pyranine. Fitting nicotinic acid quenching data by using the modified Stern-Volmer law I (Equation (3)) would result in a plot which would still curve a little bit upward which tells us how strong the static quenching of nicotinic acid is to pyranine. The higher quenching ability of nicotinic acid can be also because it does not have a benzene ring beside the amine, so less spatial hinder happened for the formation of complex between pyranine and nicotinic acid compared to tryptophan. So the complex between pyranine and nicotinic acid is more stable than the complex between pyranine and tryptophan. 


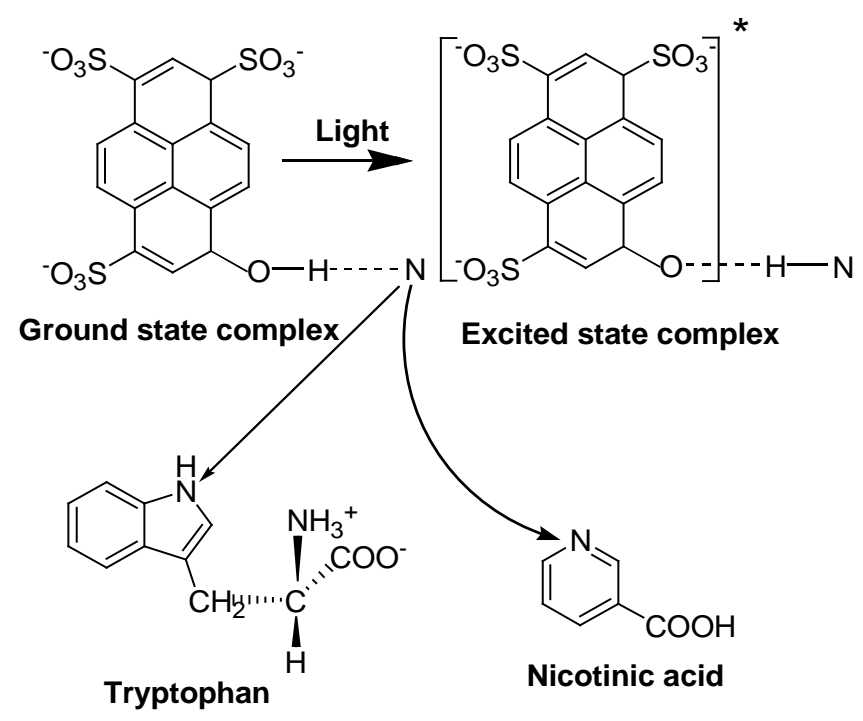

Figure 4. Scheme of the quenching mechanism of pyranine by an amine-through-hydrogen bonding complex.

There is no cyclic amine in phenylalanine, so no hydrogen bonding between pyranine and phenylalanine will form. Phenylalanine shows quenching to pyranine at steady state fluorescence but no quenching will occur dynamically. We can interpret this quenching behavior as the formation of contact complex between phenylalanine and pyranine. It is reasonable for us to infer the formation of contact complex between pyranine and phenylalanine because they both have permanent dipoles and they are both aromatic compounds. The similar benzene ring structure makes them easier to be together. We know the solubility of phenylalanine is not very high in water which means phenylalanine does not like water very much. With the addition of pyranine in the solution, phenylalanine will prefer to stay closer to pyranine. This contact complex between phenylalanine and pyranine is very weak due to the weak force between them. This contact complex can explain the quenching of phenylalanine to pyranine. As we know water is pretty good quencher by hydrogen bonding complex. Also due to its smaller size [33] [34] it is easier for water to diffuse to fluorophores to quench fluorescence. If Phenylalanine is not close enough to fluorophore, no way it can compete with water to quench the fluorescence of pyranine. So we can conclude that contact complex between pyranine and phenylalanine form at ground state which makes phenylalanine close enough to pyranine. When pyranine is excited, the larger dipole moment creates stronger interactions between phenylalanine and pyranine which we interpret as the formation of exciplex. With the formation of exciplex, the fluorescence is quenched.

By time resolved study of quenching of phenylalanine to pyranine shows phenylalanine will not quench fluorescence of pyranine dynamically which strongly support our contact complex formation. Due to the larger size of phenylalanine compared to water, it is impossible for it to diffuse to pyranine within the lifetime of fluorescence to quench the fluorescence. So no dynamic quenching will exist at all. The quenching of pyranine by phenylalanine is through the formation of contact complex between phenylalanine and pyranine. The increase of lifetime of pyranine with the increase of the concentration of phenylalanine is because the contact complex between phenylalanine and pyranine will protect pyranine quenched by water. The more phenylalanine in solutions, the more contact complexes will form. So lifetime will increase with the concentration of phenylalanine.

The absorption spectra shown in Figure 5 showed that low wavelength bands $236 \mathrm{~nm}$ and $246 \mathrm{~nm}$ were missing by introducing tryptophan, nicotinic acid and phenylalanine. Absorption bands at $282 \mathrm{~nm}$ and $291 \mathrm{~nm}$ were missing in the tryptophan and nicotinic acid. These low wavelengths bands are assigned to some structure between pyranine and water. The missing low wavelength bands are taken as the evidence of stronger interaction of pyranine with solute molecules. With the addition of solutes into solutions, the structure between pyranine and water were destroyed to different degrees due to the different interactions between pyranine and solutes. Stronger interactions will destroy more which will make four peaks disappear such as in tryptophan and nicotinic acid and in phenylalanine only two peaks will disappear which shows a weaker interaction. But the fluorophore-quencher interaction did not change the absorption and fluorescence spectral properties, because the 


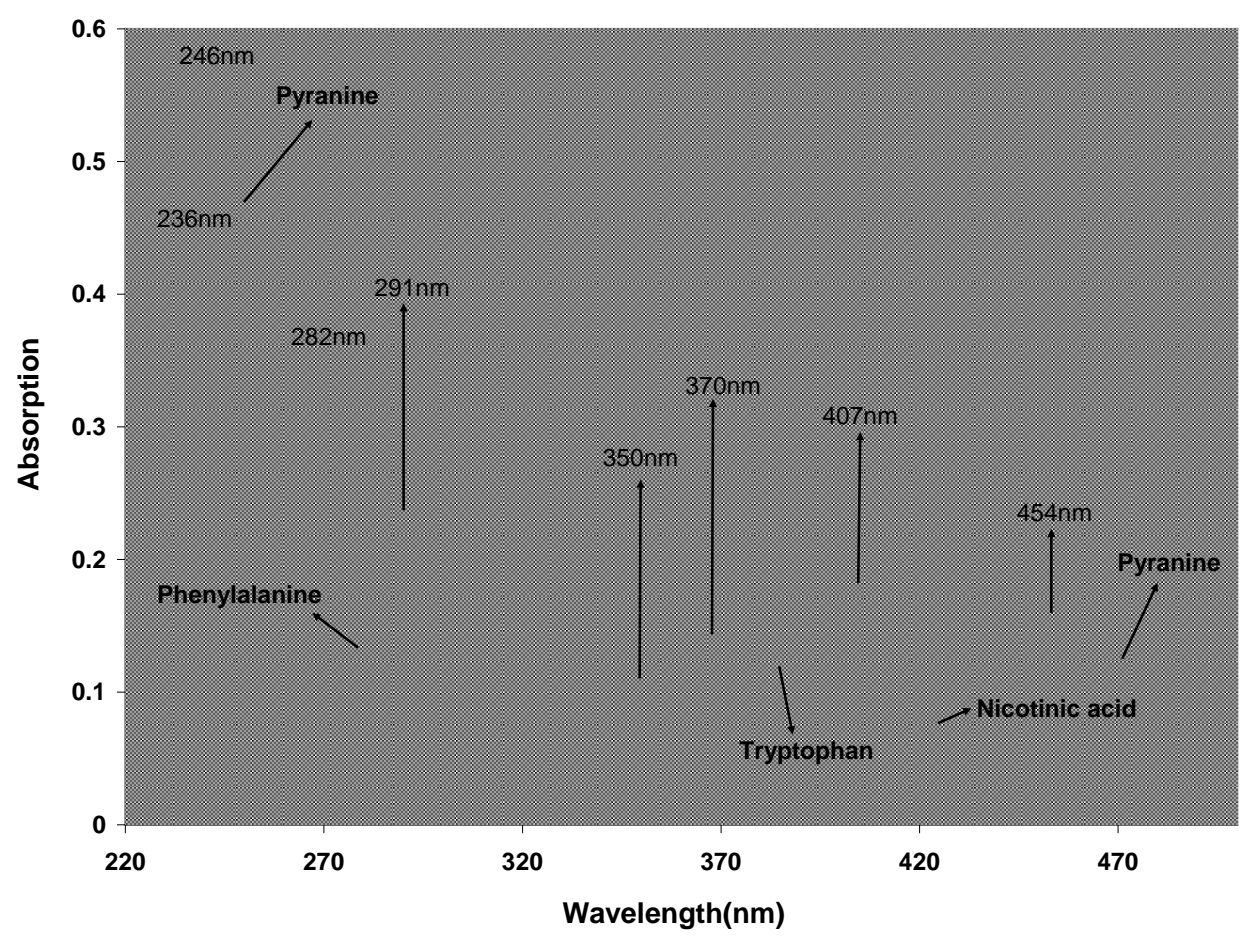

Figure 5. Absorption spectra of pyranine in water with water as reference and in tryptophan solution (Trp), nicotinic acid solution (N) and phenylalanine solution (Phe) with the solutions of the same concentration as references.

ground-state complex formed between pyranine and tryptophan or nicotinic acid were not sufficiently stable owing to weak interaction and the exciplexes are non emissive species. We do be able to assign ground state complexes because the absorption spectra bands shown in Figure 5 show a small red shift for tryptophan and nicotinic acid, and a larger red shift for nicotinic acid, which tell us the stabilization of the excited state will be improved due to the hydrogen bonding interaction which was proved by other authors too [26]. Stronger interaction between pyranine and nicotinic acid is shown by a larger red shift in the absorption spectra.

In summary when a fluorophore is dissolved in water, quenching from water will always compete with the quenching from solutes. Due to the smaller size of water it always easier to diffuse to the fluorophore and also due to the ability of formation of hydrogen bonding complex, water is always a better quencher without some other stronger interaction in the way. Solutes must have stronger interactions with the fluorophore so it can show effective quenching to fluorophores. The quenching of pyranine by solutes are the results of formation of complexes. Contact complex will form first if there is no possibility to form other types of complex. This is the case of phenylalanine quenching pyranine. The amine in the ring which is good electron donor makes the formation of hydrogen complex possible and is the reason for the stronger quenching ability of tryptophan and nicotinic acid to pyranine. So the formation of contact complex is the first step for the quenching in water solution especially for the fluorophore with functional groups that are good electron acceptors such as the hydroxyl group.

\subsection{Associates in Tryptophan and Phenylalanine Solutions}

The Stern-Volmer quenching curve of pyranine by phenylalanine at $382 \mathrm{~nm}$ is shown in Figure 6 . The quenching curve deviated downward smoothly with the concentration of phenylalanine. The quenching data were treated by the modified Stern-Volmer Law III (Equation (6)) and a straight line was obtained with intercept 1.37 and slope 1.74 shown in Figure 7. The intercept is the reciprocal of the fraction of the fluorescence accessible to the quenching. The fraction of the fluorescence accessible to the quenching is $73 \%$. This means in phenylalanine there are only $73 \%$ of phenylalanine molecules accessible to pyranine to quench fluorescence of pyranine and $27 \%$ of phenylalanine could not access pyranine at all. From the slope the quenching constant is 0.79 . From the initial slope of the quenching curve the quenching constants of pyranine by phenylalanine at $382 \mathrm{~nm}$ is 0.9 . 


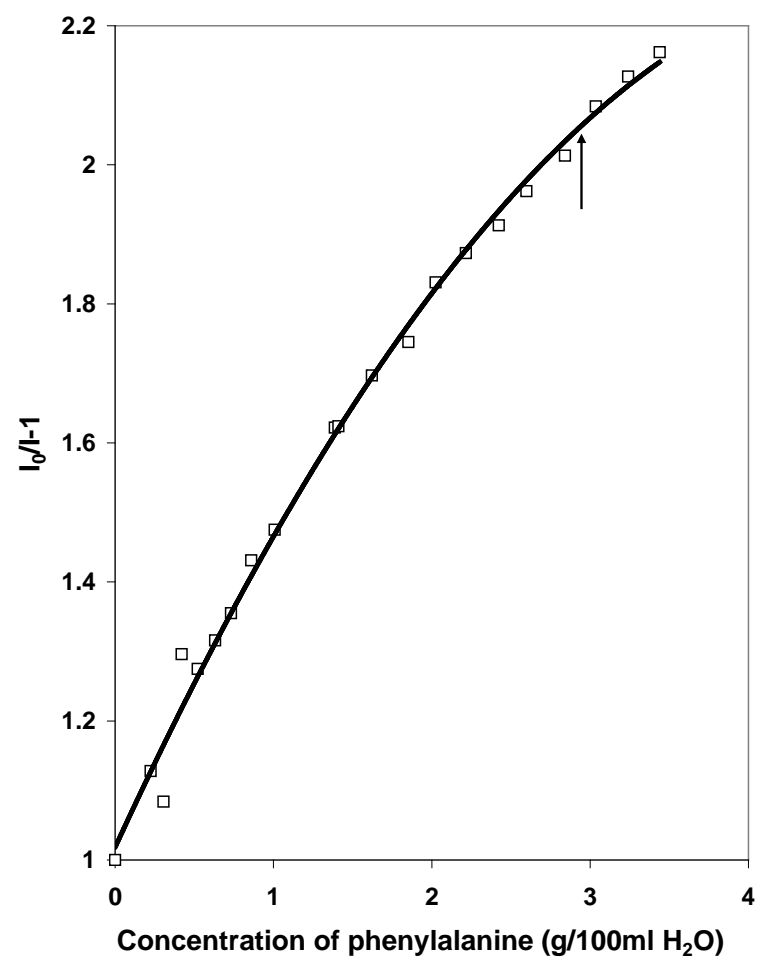

Figure 6. Quenching curve of pyranine $\left(10^{-5} \mathrm{M}\right)$ by phenylalanine at $382 \mathrm{~nm}$. The arrow shows the solubility of phenylalanine. The curve is fitted by Stern-Volmer law $I_{0} / I-1=K_{s v}[Q]$. The quenching curve of pyrnaine by phenylalanine at $382 \mathrm{~nm}$.

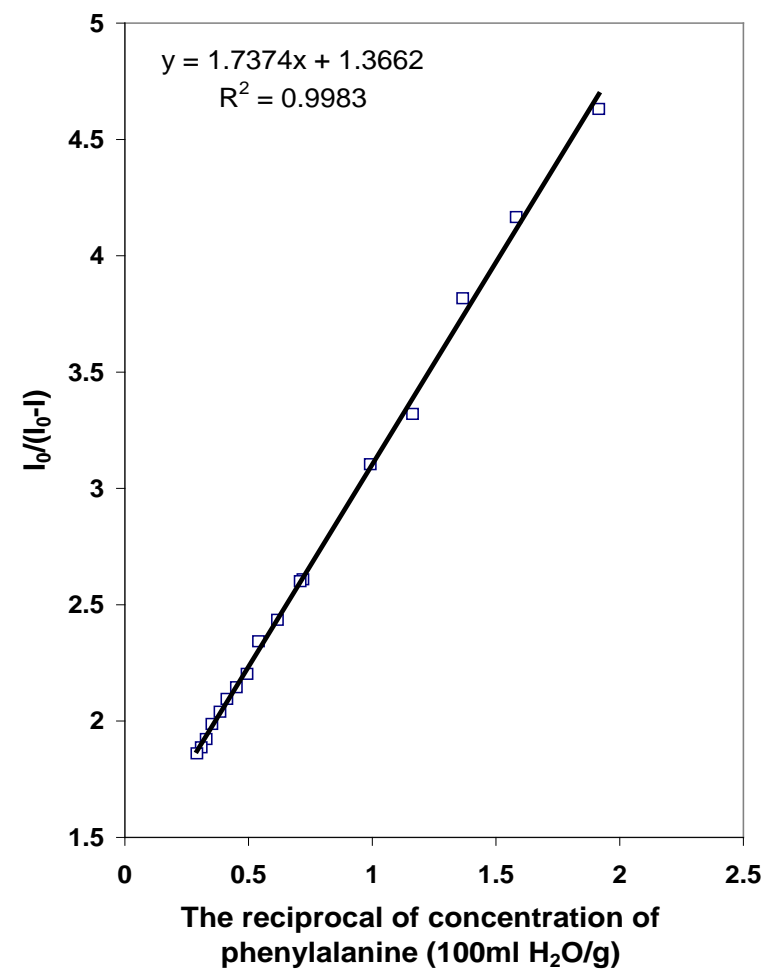

Figure 7. Quenching curve of pyranine $\left(10^{-5} \mathrm{M}\right)$ by phenylalanine at $382 \mathrm{~nm}$ fitted by Modified Stern-Volmer III (Equation (6)). 
The dynamic quenching of pyranine by tryptophan at $510 \mathrm{~nm}$ by lifetime measurement is shown in Figure 8 . The quenching curve deviated downward smoothly with the concentration of tryptophan which is not very common. We know from the previous discussion, tryptophan will quench fluorescence of pyranine by diffusion control dynamic quenching and static quenching by formation of hydrogen bonding complex. The dynamic S-V quenching curve should be a straight line, but here a downward stern-Volmer plot results.

Negative deviations from the Stern-Volmer Law are the results of multiple emitters with different quenching constants, inefficient quenching [16] [18], or many possible quenching mechanisms existing at the same time. Kikuchi and Sato [19] showed that the quenching curve of oxygen as quenchers sometimes would deviate downward from a straight line when the concentration of oxygen was high which was reported as the evidence for exciplex formation.

Negative curvatures of the plots in pyranine-tryptophan and pyranine-phenylalanine systems are likely the result of two or three dimensional structures among tryptophan and phenylalanine molecules. At $\mathrm{pH}$ range 4 - 8 the predominate species of tryptophan and phenylalanine is a zwitterion ${ }^{+} \mathrm{H}_{3} \mathrm{NRCOO}^{-}$, so the Coulomb electrostatic interaction between two or more zwitterions can form a chain or three dimensional structure.

Hydrogen bonding may be the other reason for the two or three dimensional structures. Here we call these two for three dimensional structure associates.

Due to the formation of associates among the solute molecules, the effective concentration of quenchers will not be direct proportional to true concentrations. The molecules in the middle of the associates could hardly contact with pyranine no matter by dynamic or by static quenching. Contact is required by fluorescence quenching. Associates make the quenching from some molecules impossible and also quenching by diffusion in tryptophan solutions was inhibited due to the big size of molecules. So the intensity of fluorescence will not decrease with the concentration linearly so the quenching plot curves downward. The formation of associates in tryptophan solutions among tryptophan molecules are supported by the excitation wavelength dependence of tryptophan fluorescence discussed in another paper in detail [35].

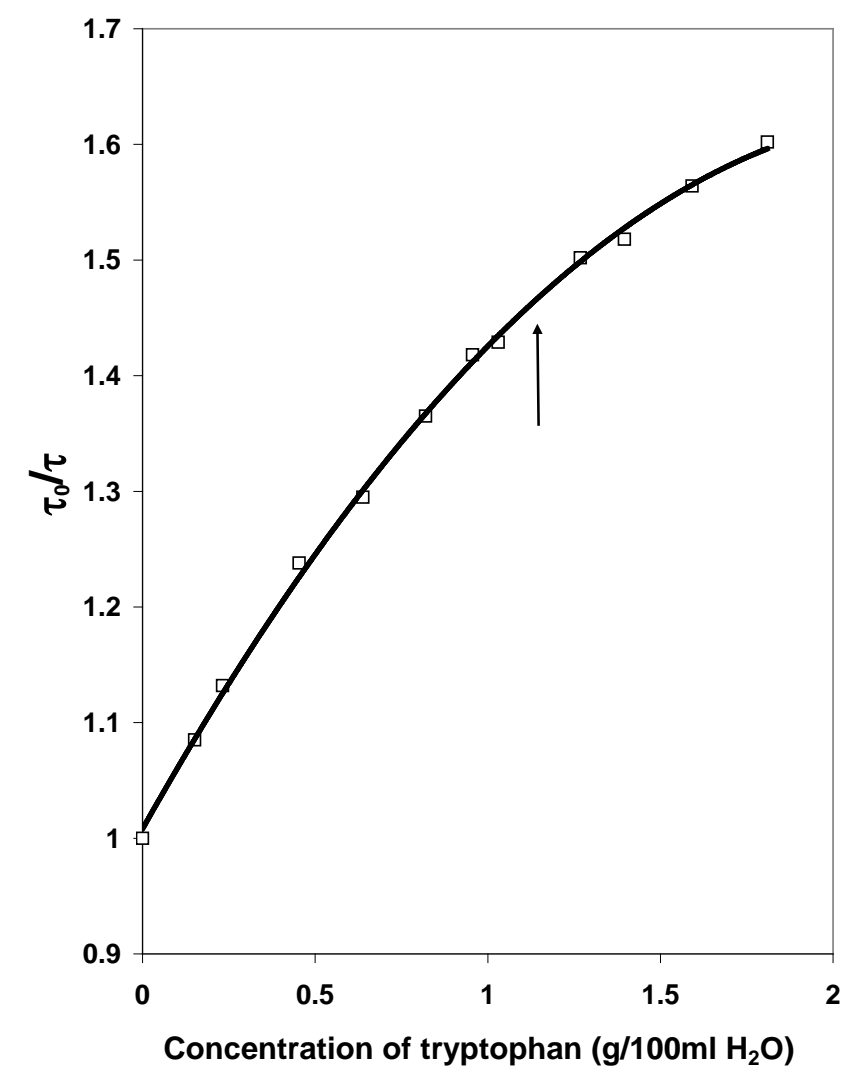

Figure 8. Lifetime S-V quenching curve of pyranine by tryptophan at $510 \mathrm{~nm}$. Arrow indicates the solubility of tryptophan. 
The higher quenching ability of nicotinic acid to pyranine compared with tryptophan is because nicotinic acid cannot form chain structures among molecules. When the concentration of nicotinic acid was low all the single molecules were good quenchers. Some which were far from fluorophores could diffuse to pyranine, meet pyranine and quench it. Other molecules could form hydrogen bonding complexes with pyranine and quench pyranine by proton transfer from excited pyranine to nicotinic acid. Both dynamic quenching and static quenching exist at the same time which results in the upward Stern-Volmer plot.

\subsection{Aggregates in Supersaturation Solutions}

Quenching of pyranine by tryptophan at $382 \mathrm{~nm}$ is shown in Figure 9. The Stern-Volmer curve of tryptophanpyranine was a straight line when the concentration of tryptophan was low. As tryptophan concentration increase a maximum is obtained and the quenching curve goes downward.

Using a ground complex model to fit the quenching data, the quenching curve of $\left(I_{0} / I-1\right) /[Q]$ versus $[Q]$ is shown in Figure 10. Before the definite concentration the quenching curve was a straight line. When the concentration is high the plots would curve downward.

In order to study the structure of supersaturated solutions, many researches have been conducted trying to find the distinguishing features of supersaturated solutions by investigating the dependence of various physical properties on concentration. The discontinuity of the property versus concentration curves at the equilibrium saturation point has rarely been observed. But in our research the quenching curves show the discontinuity at higher concentration which tells us that unsaturated and supersaturated solution show different quenching behaviors. These different behaviors can be taken as the evidence of different structure of unsaturated solution and supersaturated solution.

As we know at high concentration in high solubility systems, the observation of concentration gradient was taken as the evidence of the formation of aggregates in solution [2]. In our low solubility systems, the concentration gradient will not happen at all. Even it happens it will not be noticeable due to the low concentration. But

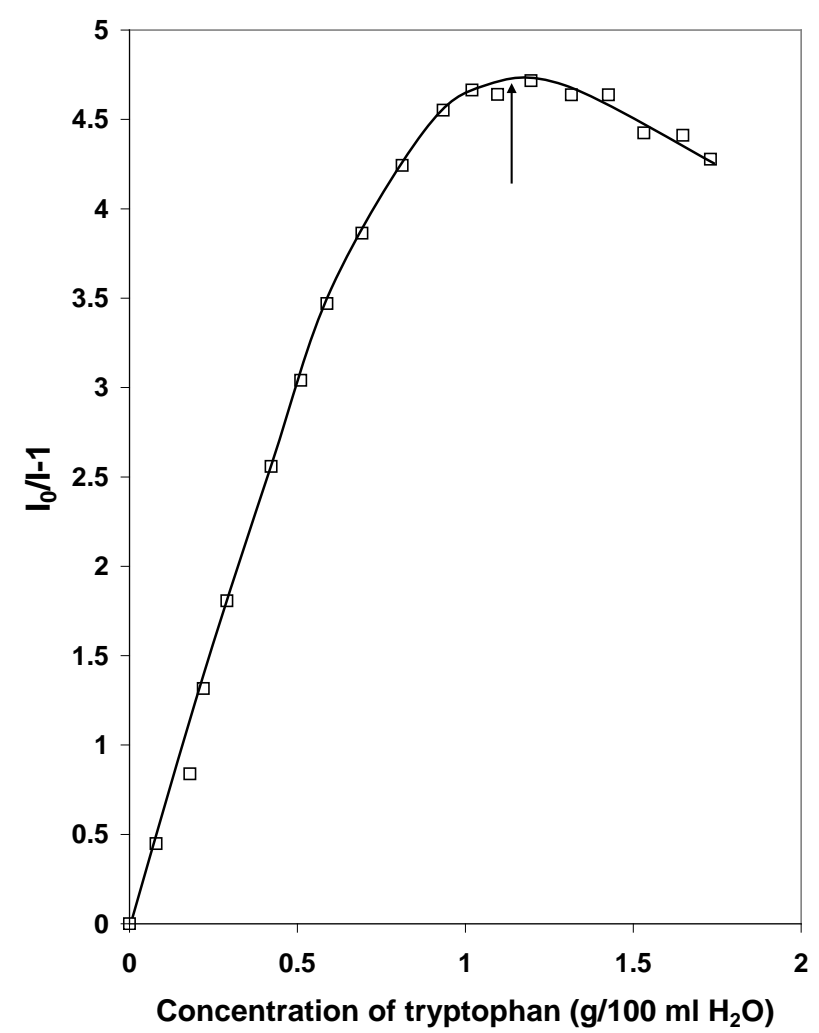

Figure 9. Quenching curve of pyranine $\left(10^{-5} \mathrm{M}\right)$ by tryptophan at $382 \mathrm{~nm}$. The curve was fitted by S-V Law $I_{0} / I-1=K_{\text {sv }}[Q]$. The arrow shows the solubility of tryptophan. The equation shown is the straight line fitting equation. 
the much lower quenching ability occurs at very high concentration which implies strongly the formation of aggregates in low solubility systems. The aggregates will have much less ability to quench fluorescence of pyranine due to its relative big size which make it very hard to diffuse to pyranine to quench fluorescence. Some molecules in the middle of aggregates cannot contact with pyranine which is the requirement for the quenching. So quenching plots will curve down and finally go downward. So we can conclude aggregates will form in the low solubility system as in the high solubility system at high concentration. Especially in tryptophan solutions, the zwitterion form will help the formation of aggregates, so the change of the quenching plots is really dramatic. At high concentration the plot will go down with the increase of concentration (Figure 9). In nicotinic acid the quenching plot will just curve downward at high concentration to indicate the formation of aggregates (Figure 10).

Fluorescence quenching technique is very easy and simple method. However, the quenching studies can be performed with a great degree of precision and get very important information about the organization of solutes.

\section{Conclusion}

Different quenching behaviors of tryptophan, phenylalanine and nicotinic acid to pyranine indicate the differences of the host-probe interaction. The differences come from different structures of tryptophan, phenylalanine and nicotinic acid. Nicotinic acid and tryptophan likely form a hydrogen bonding complex with pyranine for both ground and excited states due to the electron donor of amine. The effective quenching is the result of proton transfer from excited pyranine to amine by hydrogen bond. Contact complexes will form between phenylalanine and pyranine which is the reason for quenching of pyranine by phenylalanine. The two- or three-dimensional structures likely form in tryptophan and phenylalanine solutions due to the zwitterion ${ }^{+} \mathrm{H}_{3} \mathrm{NRCOO}^{-}$structure or/and hydrogen bonding. The aggregates are proposed in the supersaturated solutions due to the discontinuity in the quenching curves which occurs at high concentrations.

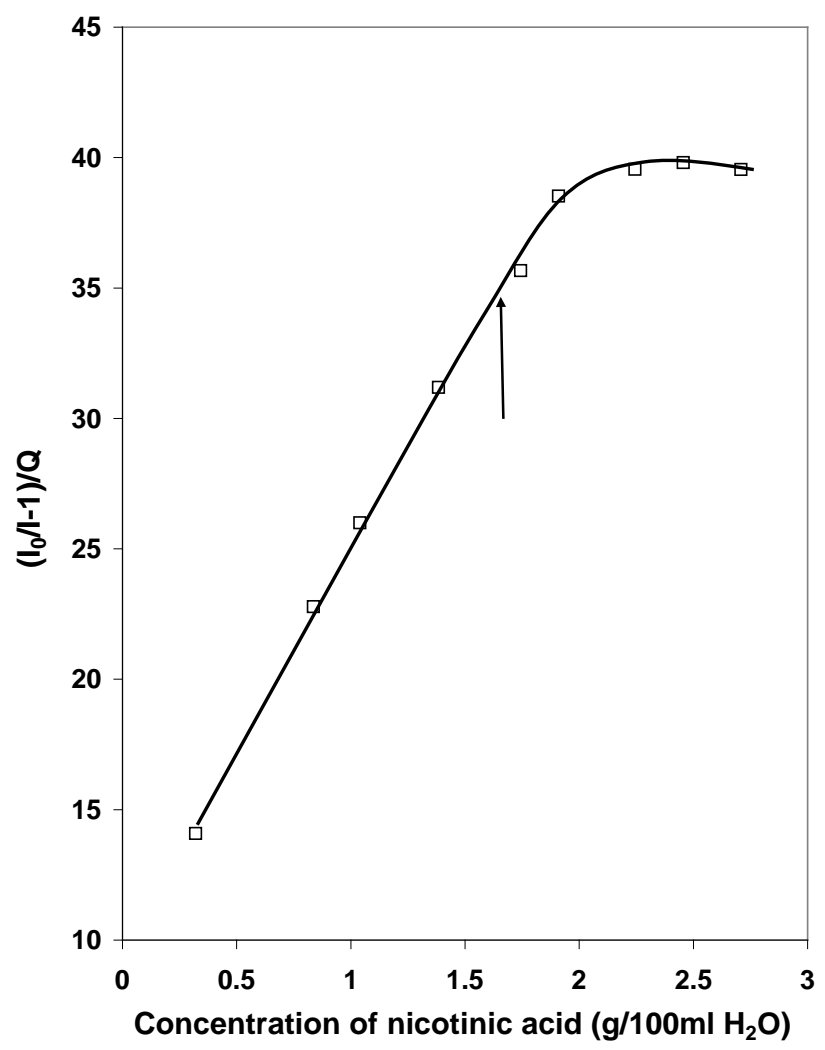

Figure 10. Quenching curve of pyranine by nicotinic acid at $382 \mathrm{~nm}$ fitted by ground state complex model $\left(I_{0} / I-1\right) /[Q]=K_{1}+K_{2}[Q]$. Arrow indicates the solubility of nicotinic acid. 


\section{Acknowledgements}

The authors acknowledge the financial support from the National Science Foundation (Grant No. NCF CTS 9407563).

\section{References}

[1] Coetzee, J.F. and Ritchie, C.D. (1969) Solute-Solvent Interactions. Marcel Dekker, New York and London

[2] Mullin, J.W. and Leci, C.L. (1969) Evidence of Molecular Cluster Formation in Supersaturated Solutions of Citric Acid. Philosophical Magazine, 19, 1075-1077.

[3] Cussler, E.L. (1980) Cluster Diffusion in Liquids. AIChE Journal, 26, 43. http://dx.doi.org/10.1002/aic.690260108

[4] Sorrel, L.S. and Myerson, A.S. (1982) Diffusivity of Urea in Concentrated, Saturated and Supersaturated Solutions. AIChE Journal, 28, 772. http://dx.doi.org/10.1002/aic.690280511

[5] Hussman, G.A., Larson, M.A. and Berglund, K.A. (1984) Industrial Crystallization'84. Jancic, S.J. and DeJong, E.J., Eds., Elsevier, Amsterdam.

[6] McMahon, P.M., Berglund, K.A. and Larson, M.A. (1984) Industrial Crystallization'84. Jancic, S.J. and DeJong, E.J. Eds., Elsevier, Amsterdam.

[7] Marcelo, H.G. and De Schryver, F.C. (1993) Time-Resolved Fluorescence Quenching in Micellar Assemblies. Chemical Reviews, 93, 199-221. http://dx.doi.org/10.1021/cr00017a010

[8] Abrams, F.S. and London, E. (1992) Calibration of the Parallax Fluorescence Quenching Method for Determination of Membrane Penetration Depth: Refinement and Comparison of Quenching by Spin-Labeled and Brominated Lipids. Biochemistry, 31, 5312-5322. http://dx.doi.org/10.1021/bi00138a010

[9] Kachel, K., Asuncion-Punzalan, E. and London, E. (1995) Anchoring of Tryptophan and Tyrosine Analogs at the Hydrocarbon-Polar Boundary in Model Membrane Vesicles. Biochemistry, 34, 15475-15479. http://dx.doi.org/10.1021/bi00047a012

[10] Peviani, C., Hillen, W., Ettner, N., Lami, H., Doglia, S.M., Piemont, E., Ellouze, C. and Chabbert, M. (1995) Spectroscopic Investigation of Tet Repressor Tryptophan-43 upon Specific and Nonspecific DNA Binding. Biochemistry, 34, 13007-13015. http://dx.doi.org/10.1021/bi00040a011

[11] Eftink, M.R. and Ghiron, C.A. (1976) Exposure of Tryptophanyl Residues in Proteins. Quantitative Determination by Fluorescence Quenching Studies. Biochemistry, 15, 672-680. http://dx.doi.org/10.1021/bi00648a035

[12] Kondo, H., Miwa, I. and Sunamoto, J. (1982) Biphasic Structure Model for Reversed Micelles. Depressed Acid Dissociation of Excited-State Pyranine in the Restricted Reaction Field. The Journal of Physical Chemistry, 86, 4826-4831. http://dx.doi.org/10.1021/j100221a035

[13] Bardez, E., Goguillon, B., Keh, E. and Valeur, B. (1984) Dynamics of Excited-State Reactions in Reversed Micelles. 1. Proton Transfer Involving a Hydrophilic Fluorescent Probe. The Journal of Physical Chemistry, 88, 1909-1913. http://dx.doi.org/10.1021/j150653a048

[14] Correll, G.D., Cheeser III, R.N., Mome, F. and Fendler, J.H. (1978) Fluorescence Probes in Reversed Micelles. Luminescence Intensities, Lifetimes, Quenching, Energy Transfer, and Depolarization of Pyrene Derivatives in Cyclohexane in the Presence of Dodecylammonium Propionate Aggregates. Journal of the American Chemical Society, 100, 12541262. http://dx.doi.org/10.1021/ja00472a037

[15] Demas, J.N. (1983) Excited State Lifetime Measurements. Academic Press, New York.

[16] Lakowicz, J.R. (1983) Principles of Fluorescence Spectroscopy. Plenum Press, New York and London.

[17] Birks, J.B. (1970) Photophysics of Aromatic Molecules. Wiley-Interscience, New York.

[18] Eftink, M.R. and Ghiron, C.A. (1981) Fluorescence Quenching Studies with Proteins. Analytical Biochemistry, 114, 199-227. http://dx.doi.org/10.1016/0003-2697(81)90474-7

[19] Kikuchi, K., Sato, C., Watabe, M., Ikeda, H., Takahashi, Y. and Miyashi, T. (1993) New Aspects of Fluorescence Quenching by Molecular Oxygen. Journal of the American Chemical Society, 115, 5180-5184. http://dx.doi.org/10.1021/ja00065a033

[20] Pan, R.-J. and Cherry, R.J. (1995) Evidence That Eosin-5-Maleimide Binds Close to the Anion Transport Site of Human Erythrocyte Band 3: A Fluorescence Quenching Study. Biochemistry, 34, 4880-4888. http://dx.doi.org/10.1021/bi00014a045

[21] Eftink, M.R. and Ghiron, C.A. (1976) Exposure of Tryptophanyl Residues in Proteins. Quantitative Determination by Fluorescence Quenching Studies. Biochemistry, 15, 672-680. http://dx.doi.org/10.1021/bi00648a035

[22] Tabak, M., Sartor, G., Neyroz, P., Spisni, A. and Canatorta, P. (1990) Interaction of Copper and Nickel Ions with 
Tryptophan and Glyciltryptophan: Time resolved Flourescence Study. Journal of Luminescence, 46, 291-299. http://dx.doi.org/10.1016/0022-2313(90)90042-A

[23] Goswami, D., Sarpal, R.S. and Dogra, S.K. (1991) Fluorescence Quenching of Few Aromatic Amines by Chlorinated Methanes. Bulletin of the Chemical Society of Japan, 64, 3137-3141. http://dx.doi.org/10.1246/bcsj.64.3137

[24] Pal, H., Palit, D.K., Mukherjee, T. and Mittal, J.P. (1990) Interaction of the Excited Singlet State of Disubstituted Anthraquinones with Aromatic Hydrocarbons: A Fluorescence-Quenching Study. Chemical Physics Letters, 173, 354359. http://dx.doi.org/10.1016/0009-2614(90)85283-I

[25] Lumry, R. and Hershberger, M. (1978) Status of Indole Photochemistry with Special Reference to Biological Applications. Photochemistry and Photobiology, 27, 819-840. http://dx.doi.org/10.1111/j.1751-1097.1978.tb07683.x

[26] Miyasaka, H., Tabata, A., Ojima, S., Ikeda, N. and Mataga, N. (1993) Femtosecond-Picosecond Laser Photolysis Studies on the Mechanisms of Fluorescence Quenching Induced by Hydrogen-Bonding Interactions-1-Pyrenol-Pyridine Systems. The Journal of Physical Chemistry, 97, 8222-8228. http://dx.doi.org/10.1021/j100133a017

[27] Mac, M., Najbar, J. and Wirz, J. (1995) Fluorescence Quenching of Derivatives of Anthracene by Organic Electron Donors and Acceptors in Acetonitrile. Electron and Proton Transfer Mechanism. Chemical Physics Letters, 235, 187194. http://dx.doi.org/10.1016/0009-2614(95)00145-T

[28] Sharma, A., Wolfbels, O.S. and Machwe, M.K. (1990) Extremely Efficient Quenching of the Fluorescence of Skatole by Pyridine. Analytica Chimica Acta, 230, 213-215. http://dx.doi.org/10.1016/S0003-2670(00)82785-3

[29] Logunov, S.L. and Rodgers, M.A. (1992) Subnanosecond Dynamics of a Naphthalene-Oxygen Exciplex. The Journal of Physical Chemistry, 96, 2915-2917. http://dx.doi.org/10.1021/j100186a025

[30] Graf, U., Niikura, H. and Hirayama, S. (1997) Fluorescence Quenching by Oxygen. Lack of Evidence for the Complex Formation of Oxygen with 9-Cyanoanthracene and Anthracene in a Supersonic Free Jet. The Journal of Physical Chemistry A, 101, 1292-1298. http://dx.doi.org/10.1021/jp962951y

[31] Talavera, E.M., Quintero, B. and Alvarez, J.M. (1992) Static and Dynamic Fluorescence Quenching of Carbazole by Tropanic Alkaloids. Journal of Photochemistry and Photobiology A: Chemistry, 66, 171-184. http://dx.doi.org/10.1016/1010-6030(92)85211-C

[32] Pal, H., Palit, D.K., Mukherjee, T. and Mittal, J.P. (1993) Dynamics of the Fluorescence Quenching of 1,4-Dihydroxy-, 1-Amino-4-Hydroxy- and 1,4-Diamino-9, 10-Anthraquinones by Aromatic Hydrocarbons. Journal of the Chemical Society, Faraday Transactions, 89, 683. http://dx.doi.org/10.1039/ft9938900683

[33] McMahon, L.P., Colucci, W.J., McLaughlin, M.L. and Barkley, M.D. (1992) Deuterium Isotope Effects in Constrained Tryptophan Derivatives: Implications for Tryptophan Photophysics. Journal of the American Chemical Society, 114, 8442-8448. http://dx.doi.org/10.1021/ja00048a014

[34] Chen, Y., Liu, B., Yu, H.-T. and Barkley, M.D. (1996) The Peptide Bond Quenches Indole Fluorescence. Journal of the American Chemical Society, 118, 9271-9278. http://dx.doi.org/10.1021/ja961307u

[35] Wang, Q.X. (1998) Fluorescence Based Sensing for Crystallization and for Characterization. Ph.D. Dissertation, Michigan State University, East Lansing, MI, USA. 